Eliana Aguiar Petri Nahas ${ }^{1}$

Bruno da Rosa de Almeida ${ }^{2}$

Daniel de Araújo Brito Buttros ${ }^{3}$

Heló́sa de Luca Véspol'

Gilberto Uemura ${ }^{1}$

JORGE NAHAS-NETO'

Artigo Original

Palavras-chave

Menopausa/metabolismo Neoplasias de mama

Síndrome X metabólica

Fatores de risco

Obesidade

Keywords

Menopause/metabolism Breast neoplasms/etiology Metabolic syndrome X Risk factors

Obesity

\title{
Síndrome metabólica em mulheres na pós-menopausa tratadas de câncer de mama
}

\author{
Metabolic syndrome in postmenopausal breast cancer survivors
}

\section{Resumo}

OBJETIVO: Avaliar a ocorrência de síndrome metabólica (SM) em mulheres na pós-menopausa tratadas de câncer de mama. MÉTODOS: Estudo clínico, transversal, com 158 mulheres na pós-menopausa (amenorreia >12 meses e idade $\geq 45$ anos) tratadas de câncer de mama e livres de doença há pelo menos cinco anos. Por meio de entrevista foram coletados dados clínicos e avaliados o índice de massa corpórea (IMC) e a circunferência da cintura (CC). Na análise bioquímica foram solicitadas dosagens de colesterol total (CT), HDL, LDL, triglicerídeos (TG), glicemia, insulina e proteína C-reativa (PCR). Foram

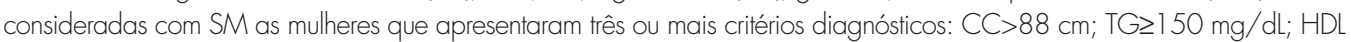
colesterol <50 mg/dL; pressão arterial $\geq 130 / 85 \mathrm{mmHg}$; glicemia de jejum $\geq 100 \mathrm{mg} / \mathrm{dL}$. Para análise estatística foram empregados o teste $t$ de Student e o teste do $\chi^{2}$. RESULTADOS: A média de idade das pacientes foi de $63,1 \pm 8,6$ anos, com tempo médio de seguimento de $9,1 \pm 4,0$ anos. A SM foi diagnosticada em 48, 1\% (76/158) e entre os critérios diagnósticos, o mais prevalente foi obesidade abdominal (CC>88 cm) afetando 54,4\% (86/158) das mulheres. As pacientes sem SM tiveram maior tempo de seguimento quando comparadas àquelas $\operatorname{com} S M(p<0,05)$. Em relação ao $I M C$ atual, aquelas sem SM eram em média sobrepeso e aquelas com SM eram obesas $(p<0,05)$. Entre estas, na comparação entre o IMC no momento do diagnóstico do câncer e o atual foi observado ganho significativo de peso $\left(27,8 \pm 5,4\right.$ versus $\left.33,4 \pm 5,4 \mathrm{~kg} / \mathrm{m}^{2}\right)$ $(p<0,05)$. O valor médio de PCR foi superior nas mulheres com SM (p<0.05). Na comparação das características tumorais e tratamentos oncológicos não houve diferença significativa entre as mulheres com e sem SM. CONCLUSÃO: Mulheres na pós-menopausa tratadas de câncer de mama têm elevado risco de desenvolver síndrome metabólica e obesidade central.

\section{Abstract}

PURPOSE: To assess the occurrence of metabolic syndrome (MełS) in postmenopausal breast cancer survivors. METHODS: A total of 158 breast cancer survivors were included in this cross-sectional study. Eligibility criteria were: women with amenorrhea $>12$ months and age $\geq 45$ years, treated for breast cancer and no metastasis for at least five years. Clinical history and anthropometric indicator data (body mass index (BMI), and waist circumference, (WC) were collected. Biochemical parameters, including total cholesterol, HDL, LDL, triglycerides (TG), glucose and C-reactive protein (CRP), were measured. MetS was diagnosed as the presence of at least three of the following diagnostic criteria: $W C>88 \mathrm{~cm}$, blood pressure $\geq 130 / 85 \mathrm{mmHg}$, triglycerides $\geq 150 \mathrm{mg} / \mathrm{dll}$, $\mathrm{HDL}<50 \mathrm{mg} / \mathrm{dL}$, and glucose $\geq 100 \mathrm{mg} / \mathrm{dL}$. The Student's ftest and $\chi^{2}$ test were used for statistical analysis. RESULTS: The mean age of breast cancer survivors was $63.1 \pm 8.6$ years, with a mean follow-up of $9.1 \pm 4.0$ years. MetS was diagnosed in $48.1 \%$ (76/158) and the most prevalent diagnostic criterion was abdominal obesity (WC>88 cm), affecting $54.4 \%$ (86/158) of the women. The patients without MetS had a longer follow-up compared those with MetS ( $p<0.05)$. Regarding the current BMI, $\mathrm{PN}$ average, those without MetS were overweight, and those with MetS were obese ( $\mathrm{p}<0.05)$. Among the latter, comparison of BMl at the time of cancer diagnosis and current BMI $\left(27.8 \pm 5.4\right.$ versus $\left.33.4 \pm 5.4 \mathrm{~kg} / \mathrm{m}^{2}\right)$ showed a significant weight gain $(p<0.05)$. Mean CRP values were higher in women with MetS ( $p<0.05)$. In the comparison of tumor characteristics and cancer treatments there was no difference between groups (p>0.05). CONCLUSION: Postmenopausal breast cancer survivors had a higher risk of developing metabolic syndrome and central obesity.

Correspondência

Eliana Aguiar Petri Nahas Departamento de Ginecologia e Obstetricica Faculdade de Medicina de Botucatu Universidade Estadual Paulisto Distrito de Rubicao Júnior $s / n^{\circ}$ CEP: 18650-000 Botucatu (SP), Brasil

Recebido 12/09/2012

Aceito com modificacōes

$08 / 10 / 2012$
Trabalho realizado no Setor de Climatério e Menopausa do Departamento de Ginecologia e Obstetrícia da Faculdade de Medicina de Botucatu da Universidade Estadual Paulista "Júlio de Mesquita Filho" - UNESP - Botucatu (SP), Brasil.

'Departamento de Ginecologia e Obstetrícia da Faculdade de Medicina de Botucatu, Universidade Estadual Paulista "Júlio de Mesquita Filho" - UNESP - Botucatu (SP), Brasil.

${ }^{2}$ Curso de Medicina de Botucatu, Universidade Estadual Paulista "Júlio de Mesquita Filho" - UNESP - Botucatu (SP), Brasil. 3Programa de Pós-graduação em Ginecologia, Obstetrícia e Mastologia da Faculdade de Medicina de Botucatu, Universidade Estadual Paulista "úlio de Mesquita Filho" - UNESP - Botucatu (SP), Brasil. 


\section{Introdução}

A sobrevida média cinco anos após diagnóstico do câncer de mama é de $61 \%$, sendo que nos países em desenvolvimento é de $57 \%$ e, nos países desenvolvidos, essa sobrevida se eleva para $73 \%{ }^{1}$. O diagnóstico precoce do câncer de mama é fundamental para que ocorra redução da mortalidade pela doença. No Brasil, o rastreamento mamográfico para mulheres entre 50 a 69 anos é estratégia recomendada para o controle desse câncer ${ }^{2} . \mathrm{Na}$ última década, o rastreamento proporcionou redução na mortalidade por câncer de mama de 1,6 a 3,4\% por ano ${ }^{3}$. Embora essa redução se deva também a tratamentos mais eficazes para o câncer de mama, muito é consequência da detecção precoce com o rastreamento populacional ${ }^{3}$. Longo tempo de seguimento é recomendado após o diagnóstico e tratamento e, com o envelhecer, o risco de comorbidades aumenta ${ }^{4,5}$. Em recente estudo, com avaliação de $91 \mathrm{mu}$ lheres na pós-menopausa em seguimento pós-tratamento do câncer de mama, houve alto índice de comorbidades ${ }^{6}$. A obesidade esteve presente em metade dos casos, hipertensão em $34 \%$, doença periférica vascular em $26 \%$ e diabetes em $13 \%$ entre as doenças mais prevalentes. Em conclusão, essa elevada taxa de comorbidades não relacionadas ao câncer pode escapar do tradicional seguimento pelo oncologista. Somente o trabalho multidisciplinar poderia oferecer qualidade na assistência a essa população ${ }^{6}$.

Estratégias atuais anticâncer têm contribuído para o aumento na sobrevida de pacientes oncológicos e em alguns casos o câncer torna-se uma doença crônica ${ }^{4,7}$. As pacientes tratadas de câncer de mama são de elevado risco para outros cânceres, assim como para doença cardiovascular (DCV), osteoporose e diabetes; sendo, portanto, reconhecidamente uma população vulnerável ${ }^{8,9}$. Assim, com o aumento da expectativa de vida das mulheres tratadas de câncer de mama, de forma semelhante àquelas sem câncer, o risco de morte por DCV aumenta ${ }^{10}$. O risco para DCV, principalmente doença coronariana, aumenta ao longo de toda a vida, com especial incremento em mulheres na pós-menopausa, quando ocupa o primeiro lugar como causa de mortalidade ${ }^{11}$.

A síndrome metabólica (SM), definida como um conjunto de fatores de riscos metabólicos que incluem obesidade abdominal, dislipidemia, hipertensão arterial e disglicemia, sabidamente associa-se com o aumento no risco de desenvolvimento de DCV e diabetes tipo $2^{12}$. Acomete aproximadamente $30 \%$ da população de mulheres com mais de $50 \operatorname{anos}^{13,14}$. Essa síndrome associa-se à desordem metabólica denominada resistência à insulina, cuja ação da insulina é prejudicada. Fatores ambientais, particularmente obesidade abdominal e inatividade física, estão amplamente implicados, mas alguns indivíduos são geneticamente predispostos à resistência a insulina ${ }^{15,16}$.
As mulheres tratadas de câncer de mama parecem apresentar risco adicional de síndrome metabólica, resultante do excesso de adiposidade e efeito dos tratamentos ${ }^{17}$. Thomson et al. ${ }^{18}$ estudaram a SM como indicador de risco para DCV em 41 mulheres na pós-menopausa com sobrepeso, tratadas de câncer de mama. Empregaram os critérios diagnósticos do US National Cholesterol Education Program: Adult Treatment Panel III (NCEP-ATP III) ${ }^{15}$, que considera a presença de pelo menos três dos seguintes critérios: obesidade abdominal (circunferência da cintura), elevação da pressão arterial, redução de HDL-colesterol, aumento de triglicerídeos e intolerância à glicose. Os autores detectaram a SM em 54,8\% das participantes, sendo a obesidade abdominal o critério mais frequente, presente em $96 \%$ dos casos, seguido pela redução do HDL em $65 \%{ }^{18}$.

Importante notar que mesmo sem as manifestações da SM, as mulheres tratadas de câncer de mama são de risco para DCV pela elevada taxa de comorbidades, como a obesidade, hipertensão e diabetes não relacionadas ao câncer ${ }^{1,18}$. Baseado nesse contexto, o objetivo do presente estudo foi avaliar a ocorrência e os indicadores de risco da síndrome metabólica em mulheres na pós-menopausa tratadas de câncer de mama.

\section{Métodos}

\section{Desenho do estudo e seleção da amostra}

Trata-se de estudo clínico, analítico e transversal. O grupo populacional foi constituído de pacientes atendidas no Centro de Avaliação em Mastologia da Faculdade de Medicina de Botucatu, de julho de 2011 a junho de 2012. O cálculo do tamanho amostral foi embasado em estudo prévio que diagnosticou SM em 54,8\% mulheres na pós-menopausa tratadas de câncer de mama ${ }^{18}$. Considerando essa frequência, com nível de significância de $5 \%$ e um erro tipo II de $10 \%$ (poder do teste de $90 \%$ ), foi estimada a necessidade de avaliar, no mínimo, 95 pacientes. Foram incluídas no estudo mulheres com: data da última menstruação há pelo menos 12 meses e idade $\geq 45$ anos; diagnóstico histológico de câncer de mama; ter completado tratamentos cirúrgico, radioterápico, hormonioterápico e quimioterápico (quando indicado); estar livre de doença há pelo menos cinco anos; não etilistas e não drogaditas. Assim, das 724 consultas médicas realizadas entre julho de 2011 a junho de 2012, 472 pacientes foram excluídas por estarem na perimenopausa, idade $<45$ anos, presença de doença metastática, menos de 5 anos de diagnóstico e recusa em participação. Foram conduzidas, portanto, 252 entrevistas. Destas, 95 não retornaram para coleta dos exames bioquímicos, portanto um total de 158 mulheres foi elegível para o estudo. Foram esclarecidos, para as pacientes selecionadas, os objetivos e procedimentos a que 
seriam submetidas, e solicitadas as assinaturas do termo de consentimento livre e esclarecido (TCLE), exigência da Resolução no 196 de outubro de 1996 do Conselho Nacional de Saúde, após aprovação pelo Comitê de Ética e Pesquisa da Faculdade de Medicina de Botucatu da Universidade Estadual Paulista "Júlio de Mesquita Filho".

\section{Metodologia}

Inicialmente, no dia da consulta, foram coletados, por meio de entrevista individual, os seguintes dados: idade, idade e tempo de menopausa, paridade, tabagismo, uso ou não de terapia hormonal (TH), história pessoal de DCV, hipertensão arterial sistêmica (HAS), diabetes, dislipidemais, atividade física e pressão arterial. Foram consideradas ativas as mulheres que praticavam exercícios físicos aeróbicos de intensidade moderada, pelo menos 30 minutos, 5 vezes na semana (150 minutos/semana) ou exercícios de resistência 3 dias por semana ${ }^{19}$. Foram diagnosticadas com síndrome metabólica as mulheres que apresentaram três ou mais critérios diagnósticos propostos pelo US National Cholesterol Education Program (NCEP)/ Adult Treatment Panel III (NCEP-ATP III $)^{15}$ : circunferência da cintura $>88 \mathrm{~cm}$; triglicerídios $\geq 150 \mathrm{mg} / \mathrm{dL}$; HDL colesterol $<50 \mathrm{mg} / \mathrm{dL}$; pressão arterial sanguínea $\geq 130 / 85 \mathrm{mmHg}$; glicemia de jejum $\geq 100 \mathrm{mg} / \mathrm{dL}$ ou sob terapia. Foram obtidos os seguintes dados para avaliação antropométrica: peso, altura, índice de massa corpórea $\left(\mathrm{IMC}=\right.$ peso/altura ${ }^{2}$ ) e circunferência da cintura (CC). Para classificação das pacientes, conforme o IMC, foi empregado o critério da World Health Organization, que considera obesidade IMC $\geq 30 \mathrm{~kg} / \mathrm{m}^{2}$. Para a medida da CC foi utilizado o ponto médio entre a última costela e a crista ilíaca, com a paciente em posição ortostática; sendo considerada aumentada para mulheres se fosse maior que $88 \mathrm{~cm}^{15}$.

A partir do levantamento de dados dos prontuários foram obtidas informações sobre o peso prévio (na ficha anestésica) e o câncer de mama quanto ao diâmetro do tumor, tipo e graduação histológica, tipo de cirurgia e estudo imunohistoquímico (receptores de estrogênio e progesterona, e Cerb-2), tempo de seguimento e tratamentos adjuvantes (radioterapia, quimioterapia e hormonioterapia). O diâmetro do tumor foi obtido a partir dos laudos histopatológicos e graduado histologicamente em graus I (bem diferenciado), II (moderadamente diferenciado) e III (indiferenciado), de acordo com método proposto que utiliza como critérios os aspectos arquiteturais, o grau de diferenciação nuclear e o índice mitótico ${ }^{20}$.

Para os exames laboratoriais, as pacientes foram orientadas a manter jejum por 12 horas. Foram realizadas avaliações do perfil lipídico e glicídico pela mensuração do colesterol total (CT), HDL, LDL, triglicerídeos (TG), e glicemia, assim como da proteína C-reativa (PCR). Todas as avaliações bioquímicas foram realizadas pelo Laboratório de Análises Clínicas da Faculdade de Medicina de Botucatu. As mensurações de triglicerídeos, CT, HDL, glicemia e PCR foram processadas pelo analisador bioquímico automático, modelo Vitros $950^{\circledR}$ pelo método colorimétrico de química seca (Johnson E Johnson, Rochester, NY, EUA). O método é linear até $800 \mathrm{mg} / \mathrm{dL}$ para TG e $900 \mathrm{mg} / \mathrm{dL}$ para CT. O LDL foi obtido subtraindo-se o valor do CT, da soma do HDL e do TG dividido por cinco. Os valores considerados ótimos foram: CT $<200 \mathrm{mg} / \mathrm{dL}, \mathrm{HDL}>50 \mathrm{mg} / \mathrm{dL}$, $\mathrm{LDL}<100 \mathrm{mg} / \mathrm{dL}, \mathrm{TG}<150 \mathrm{mg} / \mathrm{dL}$, glicemia $<100 \mathrm{mg} / \mathrm{dL}^{15}$ e PCR $<1,0 \mathrm{mg} / \mathrm{dL}^{21}$. A insulina foi quantificada pelo Sistema Immulite $^{\circledR}\left(\mathrm{DPC}^{\circledR}, \mathrm{USA}\right)$, que emprega imunoensaio, em fase sólida, por quimioluminescência, para uso em analisador automático, designado para leitura quantitativa hormonal. A taxa de normalidade para insulina, segundo o método empregado, é de 6,0 a 27,0 $\mu \mathrm{IU} / \mathrm{mL}$. Para avaliação da resistência insulínica (RI) foi utilizado método baseado em medida estática com dois constituintes plasmáticos (insulina e glicemia de jejum). $\mathrm{O}$ Homeostasis model assessment-Insulin Resistant (HOMA-IR) foi calculado pela fórmula: Insulina $\mathrm{mU} / \mathrm{mL}$ x glicemia de jejum, mmol/L/22,5. Valores que indicam RI foram definidos como Homa-IR >3, $6^{22}$.

\section{Análise estatística}

A partir dos dados foram construídas as tabelas das variáveis de mulheres na pós-menopausa tratadas de câncer de mama, quanto à presença $(n=76)$ ou ausência $(\mathrm{n}=82)$ da síndrome metabólica $(\mathrm{SM})$. Para análise dos dados foram calculados as médias e desvio padrão para as variáveis quantitativas e os percentuais para as variáveis qualitativas. $\mathrm{Na}$ comparação entre os grupos em relação às características quantitativas (clínicas, antroprométricas e bioquímicas) foi empregado o Teste $t$ de Student. $\mathrm{Na}$ associação entre a frequência das características clínicas categóricas foi empregado o teste do $\chi^{2}$. Os testes estatísticos foram bilaterais e o nível de significância adotado foi de $5 \%$. As análises foram realizadas utilizando-se Statistical Analyses System (SAS), versão 9.2, pelo Grupo de Apoio à Pesquisa (GAP) da Faculdade de Medicina de Botucatu, que deu o atendimento metodológico e conduziu os procedimentos estatísticos.

\section{Resultados}

As características clínicas e laboratoriais das 158 mulheres na pós-menopausa tratadas de câncer de mama, quanto à presença $(n=76)$ ou ausência $(n=82)$ da síndrome metabólica foram submetidas à comparação estatística, e estão representadas nas Tabelas 1 e 2. De acordo com o NCEP/ATP III, que considera a presença de três ou mais critérios para o diagnóstico, 48,1\% das mulheres foram classificadas como portadoras da síndrome metabólica. Entre 
os critérios diagnósticos, o mais prevalente foi a obesidade abdominal (CC>88cm) afetando $54,4 \%$ (86/158) das participantes. Outros critérios como hipertensão arterial, hipertrigliceridemia, baixos valores de HDL e disglicemia estiveram presentes em 50,6\% (80/158), 48,7\% (77/158), $43,7 \%$ (69/158) e 37,3\% (59/158), respectivamente.

$\mathrm{Na}$ Tabela 1 verifica-se que os grupos foram homogêneos para as seguintes variáveis: idade, tempo de menopausa, paridade, pressão arterial sistólica e diastólica e sistólica e nos valores de colesterol total e LDL ( $>>0,05)$. Observou-se diferença significante para os critérios diagnósticos da SM (CC, glicemia, HDL e TG), assim como nos valores médios do peso prévio e atual, IMC prévio e atual, insulina basal, HOMA-IR, PCR e tempo de seguimento. Na avaliação bioquímica, valores médios da PCR mostraram-se superiores nas mulheres tratadas de câncer de mama com SM, assim como apresentavam resistência a insulina demonstrado pelo HOMA-IR $(\mathrm{p}<0,05)$. As pacientes sem SM tiveram maior tempo de seguimento quando comparadas àquelas com SM. Em relação ao IMC atual, as pacientes sem SM apresentavam média de IMC de 26,9 (sobrepeso) e aquelas com SM, a média de 33,4 (obesidade) $(\mathrm{p}<0,05)$ (Tabela 1 ).

Tabela 1. Comparação entre as características clínicas e laboratoriais das 158 mulheres na pós-menopausa tratadas de câncer de mama, quanto à presença $(n=76)$ ou ausência $(\mathrm{n}=82)$ da síndrome metabólica (valores médios \pm desvio padrão)

\begin{tabular}{|c|c|c|c|}
\hline Características & Com SM (n=76) & Sem SM $(n=82)$ & Valor $p^{*}$ \\
\hline Idade (anos) & $62,4 \pm 9,2$ & $63,7 \pm 10,7$ & 0,4 \\
\hline $\begin{array}{l}\text { Idade da menopausa } \\
\text { (anos) }\end{array}$ & $48,0 \pm 4.6$ & $48,2 \pm 4,2$ & 0,7 \\
\hline $\begin{array}{l}\text { Tempo de menopausa } \\
\text { (anos) }\end{array}$ & $14,6 \pm 8,8$ & $15,1 \pm 9,3$ & 0,7 \\
\hline Paridade ( $\mathrm{n}^{0}$ de filhos) & $3,1 \pm 2,2$ & $2,7 \pm 2,0$ & 0,1 \\
\hline Peso prévio (kg) & $71,9 \pm 12,3$ & $60,4 \pm 11,2$ & $<0,001$ \\
\hline Peso atual (kg) & $76,6 \pm 12,5$ & $62,5 \pm 11,6$ & $<0,001$ \\
\hline IMC prévio (kg/m²) & $27,8 \pm 5,4$ & $25,9 \pm 4,5$ & $<0,001$ \\
\hline IMC atual $\left(\mathbf{k g} / \mathrm{m}^{2}\right)$ & $33,4 \pm 5,4$ & $26,9 \pm 4,3$ & $<0,001$ \\
\hline $\mathrm{CC}(\mathrm{cm})$ & $101,3 \pm 12,1$ & $83,9 \pm 9,6$ & $<0,001$ \\
\hline PAS (mmHg) & $139,7 \pm 21,7$ & $136,3 \pm 20,3$ & 0,307 \\
\hline PAD (mmHg) & $81,3 \pm 12,6$ & $78,1 \pm 11,2$ & 0,091 \\
\hline Glicemia (mg/dL) & $114,2 \pm 33,7$ & $90,1 \pm 14,3$ & $<0,001$ \\
\hline Insulina ( $p \mathrm{UI} / \mathrm{mL}$ ) & $18,2 \pm 11,9$ & $8,3 \pm 4,3$ & $<0,001$ \\
\hline HOMA-IR & $5,4 \pm 4,5$ & $1,9 \pm 1,2$ & $<0,001$ \\
\hline Colesterol total (mg/dL) & $211,0 \pm 43,6$ & $205 \pm 31,6$ & 0,333 \\
\hline HDL (mg/dL) & $46,9 \pm 10,5$ & $60,6 \pm 11,2$ & $<0,001$ \\
\hline LDL (mg/dL) & $124,0 \pm 37,6$ & $120,6 \pm 28,0$ & 0,5 \\
\hline Triglicerídeos (mg/dL) & $204,5 \pm 69,7$ & $115,6 \pm 36,3$ & $<0,001$ \\
\hline PCR (mg/dL) & $1,1 \pm 0,7$ & $0,7 \pm 0,6$ & 0,001 \\
\hline CA 15,3 (U/mL) & $16,8 \pm 8,3$ & $13,5 \pm 6.3$ & 0,006 \\
\hline Seguimento (anos) & $9,1 \pm 4,0$ & $11,0 \pm 5,2$ & 0,02 \\
\hline
\end{tabular}

SM: síndrome metabólica; IMC: índice de massa corporal; CC: circunferência da cintura; PAS: pressão arterial sistólica; PAD: pressão arterial diastólica; HDL: high-density lipoprotein; LDL: low-density lipoprotein; PCR: proteína reativa CA 15,3: marcador tumoral; HOMA-IR: Homeostasis model assessment-Insulin Resistant. *Diferença significativa se $\mathrm{p}<0,05$ (Teste $t$ de Student).
No grupo de pacientes com SM, na comparação entre o IMC no momento do diagnóstico do câncer e o atual $(27,8 \pm 5,4$ versus $33,4 \pm 5,4 \mathrm{~kg} / \mathrm{m}^{2}$ ) observou-se ganho significativo de peso ( $\mathrm{p}<0,001)$ no tempo médio de seguimento de $9,1 \pm 4,0$ anos. Por outro lado, entre as pacientes sem SM não houve diferença significativa nos valores médios do $\operatorname{IMC}(25,9 \pm 4,5$ versus $26,9 \pm 4,3 \mathrm{~kg} / \mathrm{m}^{2}$ ).

Quanto às características clinicas e laboratoriais, não foram observadas diferenças entre os grupos para tabagismo atual, uso prévio de $\mathrm{TH}$, atividade física e antecedente familiar de câncer de mama $(\mathrm{p}<0,05)$ (Tabela 2). Quanto ao tempo de uso prévio de TH não houve diferença entre os grupos. As pacientes com SM usaram, em média, 2,6士1,6 anos e aquelas sem $S M, 2,7 \pm 1,0$ anos $(p=0,2)$. Em relação ao IMC, maior porcentagem de pacientes com SM era obesa

Tabela 2. Associação das características clínicas e laboratoriais entre as 158 mulheres na pós-menopausa tratadas de câncer de mama, quanto à presença $(n=76)$ ou ausência ( $n=82)$ da síndrome metabólica

\begin{tabular}{|c|c|c|c|}
\hline \multirow{2}{*}{ Características } & Com SM (n=76) & Sem SM (n=82) & \multirow{2}{*}{ Valor p* } \\
\hline & $n(\%)$ & $n(\%)$ & \\
\hline Tabagismo & & & 0,2 \\
\hline Sim & $7(9,2)$ & $13(15,8)$ & \\
\hline Não & $69(90,8)$ & $69(84,2)$ & \\
\hline Uso prévio de TH & & & 0,5 \\
\hline Sim & $13(17,1)$ & $17(20,7)$ & \\
\hline Não & $63(82,9)$ & $65(79,3)$ & \\
\hline Exercício físico & & & 0,6 \\
\hline Sim & $13(17,1)$ & $12(14,6)$ & \\
\hline Não & $63(82,9)$ & $70(84,4)$ & \\
\hline AF Câncer de mama & & & 0,2 \\
\hline Sim & $36(47,4)$ & $31(37,8)$ & \\
\hline Não & $40(52,6)$ & $51(62,3)$ & \\
\hline IMC prévio (kg//m²) & & & $<0,001$ \\
\hline$\leq 24,9$ & $9(11,8)$ & $40(48,8)$ & \\
\hline $25-29,9$ & $31(40,8)$ & $29(35,4)$ & \\
\hline$\geq \mathbf{3 0}$ & $36(47,4)$ & $13(15,8)$ & \\
\hline IMC atual $\left(\mathrm{kg} / \mathrm{m}^{2}\right)$ & & & $<0,001$ \\
\hline$\leq 24,9$ & $1(1,3)$ & $33(40,2)$ & \\
\hline $25-29,9$ & $19(25,0)$ & $34(41,5)$ & \\
\hline$\geq \mathbf{3 0}$ & $56(73,7)$ & $15(18,3)$ & \\
\hline Colesterol (mg/dL) & & & 0,9 \\
\hline$<200$ & $32(42,1)$ & $35(42,7)$ & \\
\hline$\geq \mathbf{2 0 0}$ & $44(57,9)$ & $47(57,3)$ & \\
\hline LDL (mg/dL) & & & 0,5 \\
\hline$<100$ & $21(27,6)$ & $19(23,2)$ & \\
\hline$\geq 100$ & $55(72,4)$ & $63(76,8)$ & \\
\hline PCR (mg/dL) & & & $<0,001$ \\
\hline$<1,0$ & $49(64,5)$ & $74(90,2)$ & \\
\hline$\geq 1,0$ & $27(35,5)$ & $8(9,8)$ & \\
\hline
\end{tabular}

Valores expressos em número e porcentagem entre parênteses. SM: síndrome metabólica; TH: terapia hormonal; IMC: índice de massa corporal; LDL: low density lipoprotein; PCR: proteína C-reativa; AF: antecedente familiar.

*Diferença significativa se $\mathrm{p}<0,05$ (Teste do $\chi^{2}$ ). 
$(47,4 \%)$ quando comparada às mulheres sem SM $(15,8 \%)$ $(\mathrm{p}<0,05)$. Na comparação das variáveis bioquímicas, não foram observadas diferenças significativas na distribuição percentual quanto aos valores considerados ótimos de colesterol total e LDL $(\mathrm{p}>0,05)$. Por outro lado, maior porcentagem de mulheres com SM $(35,5 \%)$ apresentaram valores elevados de PCR quando comparadas àquelas sem SM (9.8\%) ( $p>0,05)$ (Tabela 2). Entre as pacientes constatou-se, pelos exames laboratoriais, que $37,3 \%$ apresentaram disglicemia e 48,7\%, hipertrigliceridemia. Em contrapartida, no relato das pacientes apenas $13,5 \%$ referiram história de diabetes e $22,1 \%$ de hipertrigliceridemia.

Na comparação das características tumorais e tratamentos oncológicos entre as pacientes com e sem SM não foram demonstradas diferenças no estadiamento, características anatomopatológicas, imunohistoquímica e tratamentos adjuvantes (Tabela 3). Observou-se que 59,5\% (94/158) das mulheres estavam no estádio II, sendo que $90,0 \%$ (142/158) com carcinoma ductal, 45,6\% (72/158) com grau histológico II e 60,1\% (95/158) com RE/RP positivo. Em relação ao tamanho do tumor, 62,7\% (99/158) tinham entre 2 e $5 \mathrm{~cm}$ e $68,3 \%$ (108/158) com axila negativa. No total de pacientes, $50,6 \%(80 / 158)$ haviam sido submetidas a mastectomia, e 79,1\% (125/158) tratadas com radioterapia, 55,7\% (88/158) com quimioterapia e $60,1 \%$ (95/158) com hormonioterapia (Tabela 3).

\section{Discussão}

A presente pesquisa demonstrou elevada frequência de síndrome metabólica entre mulheres na pós-menopausa tratadas de câncer de mama. Entre estas, o risco de desenvolver SM foi de $48,1 \%$, superior ao observado em mulheres sem câncer de mama e de mesma faixa etária e região geográfica, que foi de 39,6\% em estudo prévio $^{14}$. Dentre os critérios adotados para o diagnóstico da SM, o mais frequente entre as pacientes tratadas de câncer de mama foi obesidade central afetando $54,4 \%$ dos casos. Esses resultados estão em concordância com outros estudos $^{18,23}$. Estudo prévio detectou SM em $54,8 \%$ de 41 mulheres na pós-menopausa tratadas de câncer de mama, sendo a obesidade abdominal o critério mais frequente ${ }^{18}$. Em estudo brasileiro, avaliando 81 mulheres (idade entre 40-80 anos) com diagnóstico recente de câncer de mama comparadas a Grupo Controle de mulheres sem a doença, foi observada a ocorrência da SM em 59,2 e 37\%, respectivamente. A obesidade central $(86,4 \%)$ e a hipertensão arterial $(60,5 \%)$ foram os critérios mais prevalentes ${ }^{23}$. Por outro lado, outro estudo demonstrou prevalência de $39 \%$ da SM entre 105 mulheres na pós-menopausa com diagnóstico recente de câncer de mama ${ }^{24}$.

A obesidade abdominal e a redução do HDL colesterol estão entre os principais contribuintes para a maior prevalência da SM no sexo feminino, principalmente entre as mulheres com 45 anos ou mais de idade, revelando o possível efeito da menopausa no estabelecimento da $\mathrm{SM}^{14,25}$. Nesse contexto, está bem estabelecido o ganho de peso entre as mulheres tratadas de câncer de mama, superior a $60 \%$ em alguns estudos ${ }^{26-28}$. No presente estudo, enquanto 40,2\% do grupo de mulheres tratadas de câncer de mama sem SM tinham índice de massa corpórea adequado, entre as pacientes com SM, 25,0\% eram sobrepeso

Tabela 3. Comparação das características tumorais e tratamentos oncológicos entre as 158 mulheres na pós-menopausa tratadas de câncer de mama, quanto à presenca $(n=76)$ ou ausência ( $n=82)$ da síndrome metabólica

\begin{tabular}{|c|c|c|c|}
\hline \multirow{2}{*}{ Características } & Com SM $(n=76)$ & Sem SM $(n=82)$ & \multirow{2}{*}{ Valor $\mathrm{p}^{*}$} \\
\hline & $n(\%)$ & $n(\%)$ & \\
\hline Estadiamento & & & 0,9 \\
\hline I & $20(26,3)$ & $24(31,6)$ & \\
\hline II & $41(54,0)$ & $40(48,8)$ & \\
\hline III & $15(19,7)$ & $18(19,6)$ & \\
\hline Tipo histológico & & & 0,7 \\
\hline Ductal & $69(90,8)$ & $73(89,0)$ & \\
\hline Lobular & $7(9,2)$ & $9(11,0)$ & \\
\hline Grau histologia & & & 0,6 \\
\hline I & $20(26,3)$ & $27(32,9)$ & \\
\hline II & $36(47,4)$ & $36(46,4)$ & \\
\hline III & $19(25,0)$ & $17(20,7)$ & \\
\hline Tamanho tumoral $(\mathrm{cm})$ & & & 0,7 \\
\hline$\leq 2$ & $24(31,6)$ & $25(30,5)$ & \\
\hline$>2 \mathrm{e} \leq 5$ & $46(60,5)$ & $53(64,6)$ & \\
\hline$>5$ & $6(7,9)$ & $4(4,9)$ & \\
\hline Stafus axilar & & & 0,9 \\
\hline Negativa & $52(68,4)$ & $56(68,3)$ & \\
\hline Positiva & $24(31,6)$ & $26(31,7)$ & \\
\hline Receptores hormonais** & & & 0,7 \\
\hline RE/RP negativos & $19(25,0)$ & $18(21,9)$ & \\
\hline RE/RP positivos & $46(60,5)$ & $49(78,1)$ & \\
\hline Cerb-2 ** & & & 0,5 \\
\hline$-/+/++$ & $45(59,2)$ & $43(52,4)$ & \\
\hline +++ & $20(26,3)$ & $24(29,3)$ & \\
\hline Tipo de cirurgia & & & 0,6 \\
\hline Conservadora & $39(51,3)$ & $39(47,6)$ & \\
\hline Mastectomia & $37(48,7)$ & $43(52,4)$ & \\
\hline Radioferapia & & & 0,9 \\
\hline Não & $16(21,0)$ & $17(20,7)$ & \\
\hline $\operatorname{Sim}$ & $60(79,0)$ & $65(79,3)$ & \\
\hline Quimioterapia & & & 0,8 \\
\hline Não & $33(43,4)$ & $37(45,1)$ & \\
\hline Sim & $43(56,6)$ & $45(54,9)$ & \\
\hline Hormonioterapia & & & 0,8 \\
\hline Não & $31(40,8)$ & $32(39,0)$ & \\
\hline Sim & $45(58,2)$ & $50(61,0)$ & \\
\hline
\end{tabular}

Valores expressos em número e porcentagem entre parênteses.

SM: síndrome metabólica; RE: receptor de estrogênio; RP: receptor de progesterona.

*Diferença significativa se $\mathrm{p}<0,05$ (Teste do $\chi^{2}$ ).

* * Perfil imunoistoquímico foi realizado em 65 pacientes com SM e 67 sem SM. 
e $73,7 \%$ eram obesas. $O$ ganho de peso ocorrido entre o diagnóstico do câncer de mama e o momento atual (tempo médio de seguimento de 9,1 14,0 anos) foi em média de $4,7 \mathrm{~kg}$, repercutindo em aumento significativo no IMC. Esse resultado é semelhante ao obtido por estudo prévio que observou IMC médio de $31,4 \pm 4,2 \mathrm{~kg} / \mathrm{m}^{2}$, com ganho médio de peso em 5 anos de $9,3 \mathrm{~kg}^{18}$.

Nesta pesquisa demonstrou-se que as pacientes com SM apresentavam, em média, resistência a insulina pelo HOMA-IR. O ganho excessivo de peso relaciona-se a diversas alterações inflamatórias e metabólicas que resultam na SM, sendo elas: deposição central de gordura, resistência insulínica (RI), hipertensão arterial e dislipidemia aterogênica ${ }^{29}$. Componentes individuais da SM, assim como a própria síndrome, têm sido associados ao aumento do risco para o câncer de mama entre as mulheres na pós-menopausa ${ }^{23,30-32}$. Estudo brasileiro demonstrou maior risco para SM e hipertensão arterial entre as pacientes com câncer de mama quando comparadas ao Grupo Controle ${ }^{23}$. Estudo populacional, que comparou 3.869 mulheres suíças e italianas na pós-menopausa com câncer de mama com 4.082 mulheres na mesma faixa etária sem câncer, encontrou que os fatores de risco significativos para câncer de mama foram diabetes mellitus, hipertensão arterial, hiperlipidemia e obesidade abdominal; todos componentes da SM. Os autores concluíram que a síndrome metabólica é fator de risco independente para câncer de mama $(R R=1,7)$ e que o risco aumenta com a idade $(\mathrm{R} R=3,0)^{32}$. Em estudo caso-controle com 777 mulheres, os pesquisadores sugerem que a SM possa ser um indicador de risco para o câncer de mama em mulheres na pós-menopausa ${ }^{31}$.

Estudos atuais indicam que a SM e a obesidade, além de fatores independentes de risco para o câncer de mama ${ }^{23,30-32}$, são importantes fatores prognósticos ${ }^{5,24,33}$. $\mathrm{Na}$ presente pesquisa não foram constatadas diferenças entre as pacientes tratadas de câncer de mama com e sem $\mathrm{SM}$ em relação aos fatores preditivos e prognósticos. Isso pode ser explicado pelo fato de terem sido incluídas neste estudo mulheres sem recidiva ao longo do tratamento, sendo selecionados apenas casos de melhor prognóstico, para os quais a avaliação de risco cardiovascular se torna importante. Estudos que demonstraram associação entre a SM e fatores prognósticos para câncer de mama avaliam pacientes no momento do diagnóstico ${ }^{5,24,33}$. Contudo, na presente pesquisa mulheres sem SM tiveram maior tempo

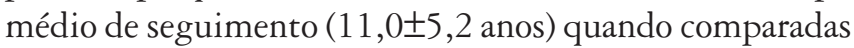
àquelas com $\mathrm{SM}(9,1 \pm 4,0$ anos). Em estudo prévio que incluiu 2.452 mulheres com história de câncer de mama em estádio inicial, os pesquisadores observaram que o diabetes tipo 2 esteve associado com pior prognóstico após 7,3 anos de seguimento 5 . Da mesma forma, em estudo avaliando 110 mulheres com câncer de mama, houve taxas mais elevadas de recorrência da doença entre as pacientes com SM após 5,5 anos de seguimento ${ }^{33}$. Em outro estudo analisando 105 mulheres na pós-menopausa com diagnóstico recente de câncer de mama, os autores observaram que as pacientes com SM, obesidade central e resistência a insulina apresentaram maior frequência de estádios II, III e IV no momento do diagnóstico quando comparadas às pacientes sem síndrome (78 e 55\% respectivamente); o alto grau histológico esteve diretamente relacionado à presença da $\mathrm{SM}^{24}$. Por outro lado, concordando com nossos resultados, em um estudo de revisão com 860 casos de câncer de mama para determinar a relação entre a presença de receptor estrogênio e a ocorrência da SM, os autores não encontraram nenhuma associação ${ }^{34}$.

Neste estudo, os valores médios de PCR mostraram-se superiores em mulheres com SM comparado aos de mulheres sem SM. Este resultado está em concordância com estudo prévio que demonstrou valores de PCR significativamente elevados entre mulheres com SM e câncer de mama ${ }^{18}$. Os valores elevados de PCR podem refletir o fato que as mulheres participantes deste estudo eram sobrepeso ou obesas, com deposição aumentada de gordura abdominal, como demonstrado em estudos prévios ${ }^{35-37}$. Elevações nos marcadores inflamatórios sistêmicos da $\mathrm{DCV}^{38-40}$ e a presença de SM $\mathrm{SM}^{33,34}$ têm sido associados com redução na sobrevida de mulheres com câncer de mama, embora o número de estudos seja limitado. Em análise de dados de uma coorte de 3.993 mulheres norte-americanas com idade entre 25 a 79 anos com diagnóstico de câncer de mama não metastático, durante um período médio de seguimento de 6,3 anos, foram verificadas 121 mortes pelo câncer e 95 mortes por $\mathrm{DCV}^{41}$. Entre as mulheres que ganharam peso pós-diagnóstico, cada $5 \mathrm{~kg}$ de ganho esteve associado a $13 \%$ de aumento no risco de morrer do câncer e a $19 \%$ de morrer por $\mathrm{DCV}^{41}$.

A avaliação da SM em mulheres na pós-menopausa tratadas de câncer de mama alerta para a necessidade de mudanças no estilo de vida visando a redução do risco de DCV, o que é relevante para a saúde dessas mulheres ${ }^{1}$. No presente estudo, entre as pacientes tratadas de câncer de mama, constatou-se pelos exames laboratoriais, que 37,3\% apresentaram disglicemia e 48,7\% hipertrigliceridemia; em contrapartida, no relato das pacientes apenas $13,5 \%$ referiram história de diabetes e $22,1 \%$ de hipertrigliceridemia. Assim, após o diagnóstico da SM, a conduta deve ser direcionada para reduzir fatores causais, como a obesidade e inatividade física, e o tratamento associado dos fatores glicídicos (disglicemia), lipídicos (dislipidemias) e não lipídicos (hipertensão arterial) ${ }^{15}$. $\mathrm{O}$ tratamento deve ser intensivo com mudança de hábitos, incluindo redução na ingestão de gordura saturada e colesterol, aumento da atividade física e controle do peso corporal ${ }^{42}$.

Entre as mulheres tratadas de câncer de mama, o ganho de peso deve-se em parte a diminuição da atividade física, associada ao estresse psicológico e físico do tratamento ${ }^{27,28,41}$. 
No presente estudo, mais de $80 \%$ das mulheres foram consideradas sedentárias, não realizando nenhum tipo de exercício físico regular. Pesquisa, avaliando o nível de atividade física antes e após 12 meses do diagnóstico do câncer de mama em 812 mulheres, encontrou diminuição da atividade física no total de 2 horas por semana. As maiores reduções foram observadas entre as pacientes tratadas com radio e quimioterapia (50\%) comparadas àquelas submetidas a cirurgia $(24 \%)$ ou a radioterapia $(23 \%)$ isoladas ${ }^{28}$. Outro estudo avaliando 753 mulheres tratadas de câncer de mama ( $>5$ anos de diagnóstico) constatou que o tempo médio referido de exercício físico moderado foi de apenas 10 minutos por semana e que somente $7 \%$ apresentaram adequado índice de alimentação saudável, repercutindo em pior qualidade de vida nesse grupo de mulheres ${ }^{9}$. Recente pesquisa, avaliando o efeito do aconselhamento nutricional por seis meses em 40 mulheres tratadas de câncer de mama sobre o peso e os índices metabólicos de risco encontrou redução média no peso de $6,1 \pm 4,8 \mathrm{~kg}$, o que repercutiu positivamente sobre os parâmetros metabólicos com melhora na relação colesterol total/HDL e diminuição nos valores de triglicerídeos e insulina ${ }^{43}$. Estudo prospectivo que incluiu 82 mulheres tratadas de câncer de mama demonstrou que a intervenção com exercício físico levou à redução dos valores de insulina e da gordura abdominal, ambos marcadores da $\mathrm{SM}^{44}$. A promoção de estilo de vida saudável é de particular importância nessa população vulnerável, incluindo a orientação alimentar e atividade física ${ }^{11,41,43}$.

Em conclusão, conforme demonstrado no presente estudo, pacientes na pós-menopausa tratadas de câncer de mama apresentam elevado risco de desenvolver síndrome metabólica e obesidade central; reconhecidamente fatores de risco para DCV e possivelmente fatores prognósticos para o câncer de mama. Assim, o seguimento dessas mulheres deveria incluir a monitorização do peso com incentivo a dieta e exercício físico e, controle da pressão arterial e do perfil lipídico e glicídico, para promover intervenção precoce e melhorar a qualidade e a sobrevida de mulheres na pós-menopausa tratadas de câncer de mama.

\section{Agradecimentos}

À Fundação de Amparo da Pesquisa do Estado de São Paulo (FAPESP), pelo apoio financeiro da Bolsa de Iniciação Científica, Processo no 2011/02459-5.

\section{Referências}

1. Redig AJ, Munshi HG. Care of the cancer survivor: metabolic syndrome after hormone-modifying therapy. Am J Med. 2010;123(1):87.e 1-6.

2. Brasil. Ministério da Saúde. Instituto Nacional de Câncer [Internet]. Tipos de câncer: mama. Rio de Janeiro: INCA; 2012 [citado 2012 Maio 5]. Disponível em: <http://www2.inca.gov.br/wps/wcm/ connect/tiposdecancer/site/home/mama>

3. American Cancer Society [Internet]. Breast cancer facts \& figures 2009-2010. Atlanta: American Cancer Society; 2009 [cited 2012 May 5]. Available from: <http://www.cancer.org/acs/groups/ content/@nho/documents/document/f861009final90809pdf.pdf>

4. Ganz PA. Survivorship: adult cancer survivors. Prim Care. 2009;36(4):721-41.

5. Patterson RE, Flatt SW, Saquib N, Rock CL, Caan BJ, Parker BA, et al. Medical comorbidities predict mortality in women with a history of early stage breast cancer. Breast Cancer Res Treat. 2010;122(3):859-65.

6. Gironés R, Torregrosa D, Díaz-Beveridge R. Comorbidity, disability and geriatric syndromes in elderly breast cancer survivors. Results of a single-center experience. Crit Rev Oncol Hematol. 2010;73(3):236-45.

7. Geiger S, Lange V, Suhl P, Heinemann V, Stemmler HJ. Anticancer therapy induced cardiotoxicity: review of the literature. Anticancer Drugs. 2010;21(6):578-90.

8. Rao AV, Demark-Wahnefried W. The older cancer survivor. Crit Rev Oncol Hematol. 2006;60(2):131-43.

9. Mosher CE, Sloane R, Morey MC, Snyder DC, Cohen HJ, Miller $P E$, et al. Associations between lifestyle factors and quality of life among older long-term breast, prostate, and colorectal cancer survivors. Cancer. 2009; 1 15(17):4001-9.

10. Ewer MS, Glück S. A woman's Heart. A woman's heart: the impact of adjuvant endocrine therapy on cardiovascular health. Cancer. 2009; 11 15(9): 1813-26.

11. Mosca L, Banka CL, Benjamin EJ, Berra K, Bushnell C, Dolor RJ, et al. Evidence-based guidelines for cardiovascular disease prevention in women: 2007 update. Circulation. 2007; 1 15(11):1481-501.

12. Schneider JG, Tompkins C, Blumenthal RS, Mora S. The metabolic syndrome in women. Cardiol Rev. 2006;14(6):286-91.

13. Ford ES, Giles WH, Mokdad AH. Increasing prevalence of the metabolic syndrome among U.S. adults. Diabetes Care. 2004;27(10);2444-9.

14. Petri Nahas EA, Padoani NP, Nahas-Neto J, Orsatti FL, Tardivo $A P$, Dias R. Metabolic syndrome and its associated risk factors in Brazilian postmenopausal women. Climacteric. 2009;12(5):43 1-8.

15. Expert Panel on the Detection, Evaluation, and Treatment of High Blood Cholesterol in Adults. Executive summary of the Third Report of the National Cholesterol Education Program (NCEP). Adult Treatment Panel III (ATP III). JAMA. $2001 ; 285(19): 2486-97$.

16. Millen BE, Pencina M, Kimokoti RW, Zhu L, Meigs JB, Ordovas $J M$, et al. Nutritional risk and the metabolic syndrome in women: opportunities for preventive intervention from the Framingham Nutrition Study. Am J Clin Nutr. 2006;84(2):434-41.

17. Rose DP, Komninou D, Stephenson GD. Obesity, adipocytokines, and insulin resistance in breast cancer. Obes Rev. 2004;5(3):153-65. 
18. Thomson CA, Thompson PA, Wright-Bea J, Nardi E, Frey GR, Stopeck A. Metabolic syndrome and elevated $C$-reactive protein in breast cancer survivors on adjuvant hormone therapy. J Womens Health (Larchmt). 2009; 18(12):2041-7.

19. Centers for Disease Control and Prevention [Internet]. Physical activity: how much physical activity to adults need? Atlanta: CDC; 2011 [cited 2012 May 5]. Available from: <http://www.cdc. gov/physicalactivity/everyone/guidelines/adults.html>

20. Elston EW, Ellis IO. Method for grading breast cancer. J Clin Pathol. 1993;46(2):189-90.

21. Ridker PM. Clinical application of C-reactive protein for cardiovascular disease detection and prevention. Circulation. 2003;107(3):363-9.

22. Stern SE, Williams K, Ferrannini E, DeFronzo RA, Bogardus C, Stern MP. Identification of individuals with insulin resistance using routine clinical measurements. Diabetes. 2005;54(2):333-9.

23. Porto LA, Lora KJ, Soares JC, Costa LO. Metabolic syndrome is an independent risk factor for breast cancer. Arch Gynecol Obstet. 2011 ;284(5):1271-6.

24. Healy LA, Ryan AM, Carroll P, Ennis D, Crowley V, Boyle T, et al. Metabolic syndrome, central obesity and insulin resistance are associated with adverse pathological features in postmenopausal breast cancer. Clin Oncol (R Coll Radiol). 2010;22(4):281-8.

25. Mesch VR, Boero LE, Siseles NO, Royer M, Prada M, Sayegh F, et al. Metabolic syndrome throughout the menopausal transition: influence of age and menopausal status. Climacteric. 2006;9(1):40-8.

26. Rock CL, Flatt SW, Newman V, Caan BJ, Haan MN, Stefanick $\mathrm{ML}$, et al. Factors associated with weight gain in women after diagnosis of breast cancer. Women's Healthy Eating and Living Study Group. J Am Diet Assoc. 1999;99(10):1212-21.

27. Chlebowoski RT, Aiello E, McTiernan A. Weight loss in breast cancer patient management. J Clin Oncol. 2002;20(4): 11 28-43.

28. Irwin ML, Crumley D, McTiernan A, Bernstein L, Baumgartner R, Gilliland FD, et al. Physical activity levels before and after a diagnosis of breast carcinoma: the Health, Eating, Activity, and Lifestyle (HEAL) Study. Cancer. 2003; 97(7): 1746-57.

29. Alberti KG, Eckel RH, Grundy SM, Zimmet PZ, Cleeman Jl, Donato $K A$, et al. Harmonizing the metabolic syndrome: a joint interim statement of the International Diabetes Federation Task Force on Epidemiology and Prevention; National Heart, Lung, and Blood Institute; American Heart Association; World Heart Federation; International Atherosclerosis Society; and International Association for the Study of Obesity. Circulation. 2009;120(16):1640-5

30. Bjørge T, Lukanova A, Jonsson $\mathrm{H}$, Tretli S, Ulmer $\mathrm{H}$, Manjer J, et al. Metabolic syndrome and breast cancer in the me-can (metabolic syndrome and cancer) project. Cancer Epidemiol Biomarkers Prev. 2010;19(7):1737-45.

31. Capasso I, Esposito E, Pentimalli F, Crispo A, Montella M, Grimaldi $M$, et al. Metabolic syndrome affects breast cancer risk in postmenopausal women: National Cancer Institute of Naples experience. Cancer Biol Ther. $2011 ; 10(12): 1240-3$.
32. Rosato V, Bosetti C, Talamini R, Levi F, Montella M, Giacosa A, et al. Metabolic syndrome and the risk of breast cancer in postmenopausal women. Ann Oncol. 201 1;22(12):2687-92.

33. Pasanisi P, Berrino F, De Petris M, Venturelli E, Mastroianni A, Panico S. Metabolic syndrome as a prognostic factor for breast cancer recurrences. Int J Cancer. 2006; $119(1): 236-8$.

34. Colonna SV, Douglas Case L, Lawrence JA. A retrospective review of the metabolic syndrome in women diagnosed with breast cancer and correlation with estrogen receptor. Breast Cancer Res Treat. 2012;131(1):325-31.

35. Fairey AS, Courneya KS, Field CJ, Bell GJ, Jones LW, Martin $B S$, et al. Effect of exercise training on C-reactive protein in postmenopausal breast cancer survivors: a randomized controlled trial. Brain Behav Immun. 2005;19(5):381-8

36. Takkouche B, Regueira-Méndez C, Etminan M. Breast cancer and use of nonsteroidal anti-inflammatory drugs: a meta-analysis. J Natl Cancer Inst. 2008;100(20): 1439-47.

37. Pierce BL, Neuhouser ML, Wener MH, Bernstein L, Baumgartner $\mathrm{RN}$, Ballard-Barbash $\mathrm{R}$, et al. Correlates of circulating C-reactive protein and serum amyloid $A$ concentrations in breast cancer survivors. Breast Cancer Res Treat. 2009;1 14(1):155-67.

38. McMillan DC, Elahi MM, Sattar N, Angerson WJ, Johnstone J, McArdle CS. Measurement of the systemic inflammatory response predicts cancer-specific and non-cancer survival in patients with cancer. Nutr Cancer. 2001;41(1-2):64-9.

39. Al Murri AM, Wilson C, Lannigan A, Doughty JC, Angerdson WJ, McArdle CS, et al. Evaluation of the relationship between the systemic inflammatory response and cancer-specific survival in patients with primary operable breast cancer. Br J Cancer. 2007;96(6):891-5.

40. Heikkilä K, Ebrahim S, Rumley A, Lowe G, Lawlor DA. Associations of circulating $C$-reactive protein and interleukin-6 with survival in women with and without cancer: findings from the British Women's Heart and Health Study. Cancer Epidemiol Biomarkers Prev. 2007; 16(6): 1 155-9.

41. Nichols HB, Trentham-Dietz A, Egan KM, Titus-Ernstoff L, Holmes $M D$, Bersch $A \mathrm{~J}$, et al. Body mass index before and after breast cancer diagnosis: associations with all-cause, breast cancer, and cardiovascular disease mortality. Cancer Epidemiol Biomarkers Prev. 2009; 18(5): 1403-9.

42. Grundy SM, Hansen B, Smith SC Jr, Cleeman JI, Kahn RA. Clinical management of metabolic syndrome: report of the American Heart Association/National Heart, Lung and Blood Institute/American Diabetes Association conference on scientific issues related to management. Circulation. 2004;109(4):551-6.

43. Thomson CA, Stopeck AT, Bea JW, Cussler E, Nardi E, Frey G, et al. Changes in body weight and metabolic indexes in overweight breast cancer survivors enrolled in a randomized trial of low-fat vs. reduced carbohydrate diets. Nutr Cancer. 2010;62(8): 1 142-52.

44. Ligibel JA, Campbell N, Partridge A, Chen WY, Salinardi T, Chen H, et al. Impact f a mixed strength and endurance exercise intervention on insulin levels in breast cancer survivors. J Clin Oncol. 2008;26(6):907-12. 